www.jmscr.igmpublication.org

Impact Factor (SJIF): 6.379

Index Copernicus Value: 71.58

ISSN (e)-2347-176x ISSN (p) 2455-0450

crossref DOI: https://dx.doi.org/10.18535/jmscr/v6i5.93

Journal Of Medical Science And Clinical Research

\title{
64 Slice CT Evaluation of Anatomical Variations of Main Arteries Arising from the Abdominal Aorta and their Branching Pattern
}

\author{
Authors \\ Gehlot Ramanand ${ }^{1}$, Chouhan Ajay Singh ${ }^{2}$, Chaturvedy Kirti Rana ${ }^{3}$, Kumar Ramesh ${ }^{4}$, \\ Sunia Inderjeet Singh ${ }^{5}$ \\ ${ }^{1}$ Professorand Head, ${ }^{2}$ Resident, ${ }^{3}$ Professor, ${ }^{4-5}$ Residents \\ Dr. S. N. Medical College and Attached Group of Hospitals, Jodhpur, India
}

\begin{abstract}
Aim: To determine the comprehensive spectrum of celiac axis including SMA, IMA, hepatic and renal artery variations with the use of multi-detector computed tomography (MDCT).

Material and Methods: This retrospective and prospective study was conducted in the Department of Radio diagnosis, Dr. S.N. Medical College and Associated Group of Hospitals, Jodhpur in 500 patients subjected to MDCT abdomen for various indications. Patients with a history of prior major upper abdominal surgery and patients with occlusion of celiac, hepatic, SMA and renal arteries were excluded.

Results: Seven types of celiac axis anatomic variations were identified in our study (including SMA). Anatomic variations in celiac axis were seen in $9 \%$ of patients with ambiguous celiac axis anatomy in $2 \%$ of the patients. IMA origin was normal in all patients. CHA originated from celiac axis in $95.20 \%$ of the patients. Variations in anatomic origin of CHA were seen in $2.8 \%$ patients. Normal origin of RHA from HAP and CHA was seen in $77.75 \%$ patients. LHA originated from HAP and CHA in $77.75 \%$ patients. MHA originated from RHA in 48\% patients, LHA in 12\% and from CHA in 12\% cases. Origin of MHA could not be defined in $28 \%$ of patients. GDA originated from CHA in $95 \%$ of patients. Single renal artery was seen in $53 \%$ patients. $49 \%$ patient have variation in the form of early branching and additional renal arteries.

Conclusion: Variations in the celiac trunk, SMA, hepatic artery and renal artery are common, and their detection is important prior to any interventions or abdominal surgeries.

Keywords: SMA/IMA-superior/inferior mesenteric artery; CHA- common hepatic artery; HAP-hepatic artery proper; RHA/LHA/MHA-right/left/middle hepatic artery; GDA-gastro duodenal artery.
\end{abstract}

\section{Introduction}

Evaluation of arteries branching from the abdominal aorta (the level of their divergence, presence of atypical variants of a common origin of arteries or presence of additional arteries) plays an important role in the surgical planning. Due to short time of the examination, the Multi detector Computed Tomography (MDCT) proves to be useful in emergency cases as well as in a quick assessment of the vascular axis and for the purposes of immediate surgeries or endovascular interventions. ${ }^{[1]}$

In 1955, Michel described the classification scheme for describing anatomic variation in the hepatic arterial blood supply based on the results of dissecting 200 cadavers [Table 1]. ${ }^{[2]}$ In 1994, 
Hiatt described surgical anatomy of the hepatic arteries in 1000 cases [Table 1]. ${ }^{[3]}$ In 1969, Vandamme et al., did extensive research on hepatic artery anomalies on 156 cadavers. ${ }^{[4]}$ Then, in 1971, Suzuki et al., published their series on 200 patients based on angiography study and highlighted the importance of hepatic artery variations. ${ }^{[5]}$ In 2010, Song et al., published the largest series of their work in celiac axis and hepatic artery variations in 5002 patients. ${ }^{[6]}$ Anatomical variations of the celiac axis and hepatic artery are described primarily according to Song's nomenclature (Table 2) and compared according to Uflacker's system ${ }^{[7]}$ for celiac axis and Michel and Hiatt classification for hepatic arteries.

Recently, with the advent of newer interventional and surgical options for patients with primary and metastatic hepatic malignancies, partial hepatectomy for liver transplantation and laparoscopic cholecystectomy, surgeons and interventional radiologists are now relying on accurate imaging and assessment of the hepatic arterial supply. A road map of the arterial vascularity of the donor and recipient is a prerequisite for transplant surgery. ${ }^{[8]}$ Detailed hepatic arterial anatomy and its variations has its significance in liver surgeries and interventional hepatic procedures, relative to the hepatic lobe involved. The RHA, being an end artery is important in hepatobiliary surgery. It is to be preserved as injury to it cause necrosis of right lobe of the liver. Presence of accessory hepatic arteries have significance in liver transplant recipient patients, these patients often have small caliber CHA which increases risk of posttransplantation hepatic artery complications like stenosis and thrombosis. ${ }^{[8]}$

Renal artery variations are not uncommon either and give rise to several problems that are encountered by clinicians. Kidneys with large number of renal arteries are reported to have a higher rate of transplantation failure than those with a single renal artery ${ }^{[9,10]}$. The risk represented by these vascular variations is not, however, limited to renal transplantations and to the surgical treatment of renovascular hypertension. Variations in the origin, course and branching pattern of the renal artery occur frequently and are of special interest to the urologists, nephrologists, surgeons and radiologists, with respect to the diseases associated with it.

Knowledge of distance of branching of renal artery from aorta is important. The one ostium usually can be obtained during laparoscopic donor nephrectomy if the early branches are beyond 10 $\mathrm{mm}$ from the origin of the main renal artery. Otherwise, the renal arteries have to be reconstructed on the back table or separate renal artery anastomosis to the recipient has to be performed. An accessory artery in the inferior renal pole crosses the ureter obliquely from its anterior aspect, and may lead to hydronephrosis by compressing the ureter. Around $70 \%$ of individuals the kidney is supplied by single renal artery arising from abdominal aorta. ${ }^{[11]}$ However, renal artery variations are very common regarding their origin and number that have been reported by many researchers. ${ }^{[12-14]}$ The renal arteries may vary in their level of origin, caliber, obliquity, number and precise relation. The frequencies of renal artery variations showed social, ethnic and racial differences ${ }^{[15]}$ for example variations in renal artery and its branching pattern are more common in Africans and less common in Indians. [16] 
Table 1: Hepatic artery variations: Michel's and Hiatt's classifications. ${ }^{[2,3]}$

\begin{tabular}{|l|l|l|l|}
\hline \multicolumn{2}{|l|}{ Michel's classification } & Hiatt classification \\
\hline Type & Frequency $(\%)$ & Description & Type \\
\hline I & 55 & $\begin{array}{l}\text { Hepatic artery originates from the CHA and } \\
\text { bifurcates into the RHA and LHA }\end{array}$ & Type I \\
\hline II & 10 & Replaced LHA arising from the LGA & Type II \\
\hline III & 11 & Replaced RHA arising from the SMA & Type III \\
\hline IV & 1 & Replaced RHA and LHA & Type IV \\
\hline V & 8 & Accessory LHA arising from LGA & Type II \\
\hline VI & 7 & Accessory RHA arising from SMA & Type III \\
\hline VII & 1 & Accessory RHA and LHA & Type IV \\
\hline VIII & 4 & $\begin{array}{l}\text { Replaced RHA and accessory LHA or replaced LHA } \\
\text { and accessory RHA }\end{array}$ & Type IV \\
\hline IX & 4.5 & Entire hepatic trunk arises from the SMA & Type V \\
\hline $\mathbf{X}$ & 0.5 & Entire hepatic trunk arises from the LGA & NOD \\
\hline NOD & & $\begin{array}{l}\text { Common hepatic artery directly originating from the } \\
\text { aorta }\end{array}$ & Type VI \\
\hline
\end{tabular}

Table 2: Celiac axis variations according to Song's classification ${ }^{[6]}$ scheme

\begin{tabular}{|l|c|}
\hline Description & $\begin{array}{c}\text { Song's classification scheme } \\
\text { (including celiac trunk and SMA) }\end{array}$ \\
\hline The CHA, SpA and LGA originating from the celiac trunk & Normal anatomy \\
\hline The CHA, SpA and LGA have a common point of origin from the celiac trunk & HGSp+SM \\
\hline $\begin{array}{l}\text { CHA and SpA have a common point of origin with the LGA demonstrates } \\
\text { variable points of origin }\end{array}$ & HSp trunk + LG + SM \\
\hline CHA and SA have common trunk with the LGA arises separately from aorta \\
\hline $\begin{array}{l}\text { CHA and LGA have common trunk with the SpA and SMA arises separately } \\
\text { from the aorta }\end{array}$ & HG trunk + Sp + SM \\
\hline $\begin{array}{l}\text { CHA, SA and SMA have common trunk with the LGA arises separately from the } \\
\text { aorta }\end{array}$ & HSpM trunk + LG \\
\hline $\begin{array}{l}\text { LGA and SA have a common trunk with the CHA and SMA arises separately } \\
\text { from the aorta or common SMA and CHA trunk from aorta }\end{array}$ & CH + GSp trunk + SM \\
\hline Celiac and SMA have a common trunk & HM trunk + GSp trunk \\
\hline The middle colic artery and the celiac have the same trunk & CM trunk \\
\hline No celiac trunk with the CHA, SpA, LG and LGA arises directly from the aorta & CH + LG + Sp + SM \\
\hline $\begin{array}{l}\text { CHA and SMA have a common trunk with SpA and LGA arise separately from } \\
\text { the aorta }\end{array}$ & HM trunk + LG + Sp \\
\hline $\begin{array}{l}\text { CHA and LGA have a common trunk similarly SMA and SpA arise as common } \\
\text { trunk from the aorta }\end{array}$ & HG trunk + SpM trunk \\
\hline $\begin{array}{l}\text { LGA, SpA and SMA have a common trunk with CHA arise separately from the } \\
\text { aorta }\end{array}$ & CH + GSpM trunk \\
\hline $\begin{array}{l}\text { SpA and SMA have a common trunk with CHA and LGA arise separately from } \\
\text { the aorta }\end{array}$ & CH + LG + SpM trunk \\
\hline $\begin{array}{l}\text { CHA and SpA have a common trunk similarly LGA and SMA arise as common } \\
\text { trunk from the aorta }\end{array}$ & HSp trunk + GM trunk \\
\hline $\begin{array}{l}\text { CHA, LGA and SMA have a common trunk with SpA arise separately from the } \\
\text { aorta }\end{array}$ & HGM trunk + Sp \\
\hline $\begin{array}{l}\text { LGA and SMA have a common trunk with CHA and SpA arise separately from } \\
\text { the aorta }\end{array}$ & CH + GM trunk + Sp \\
\hline $\begin{array}{l}\text { Absent CHAorVariant CHAs with an unclear origin due to the presence of a } \\
\text { persistent anastomotic channel }\end{array}$ & Ambiguous anatomy \\
\hline
\end{tabular}

\section{Material and Methods}

\section{Source of Data}

This retrospective and prospective hospital based study was conducted in the Department of Radio diagnosis, Dr. S. N. Medical College and Associated Group of Hospitals, Jodhpur. The necessary permission and approval from Ethics Committee and authority prior to initiation of the 
study was taken.The study population included CT images of 500 patients who underwent MDCT abdomen in our hospital for various indications between January 2017 to November 2017. The celiac trunk, SMA, IMA, hepatic and renal arterial system were individually assessed, and variations were noted.

\section{Method of Collection of Data}

The examinations were carried out with PHILIPS 64 slice CT SCANNER with Philips windows workstation and software. The examined area was stretched from the diaphragm domes to the pubic bone. Contrast agent bolus was administrated using an automatic injector. The volume of the non-ionic iodinated contrast agent ranged from 80 to $130 \mathrm{ml}$, depending on the patient's body mass. The rate of the contrast agent administration was $3.0-5.5 \mathrm{ml} / \mathrm{s}$. Contrast medium administration was followed by injection of $40 \mathrm{ml}$ of a normal saline (wash out bolus). CT examinations was performed according to two protocols: a regular CTA (onephase examination) or an abdominal CT (multiphase examination), depending on indications. The multi-phase examinations were evaluated in the early arterial phase only.

\section{Image Interpretation}

The obtained scans were analyzed using Philips windows workstation and Philips IntelliSpace portal software. Image post processing techniques involved isotropic multiplanar two- and threedimensional reconstructions (Maximum Intensity Projection MIP; Volume Rendering VR). Only those images that were free from artefacts i.e. where the arterial phase was appropriately visualized and an adequate and comprehensive evaluation of the aortic branches were possible were used for the analysis. The images were analyzed independently by three radiologists Radiologist 1 (A.S.C) with 1.5 years, Radiologist 2 (R.G.) with 25 years and Radiologist 3 (K.R.C) with 20 years of experience.
Interpretation of variation in origin of LG, SpA, SMA and origin/course of CHA

To describe the results of systematic analysis of the celiac axis including SMA and the hepatic anatomy comprehensively, we used nomenclature system described by Song et al (Table 2-4). ${ }^{[6]}$ The abbreviations and terms used in this system are listed in Tables 2-5. By integrating the data obtained from the analysis of the CT images, we classified the variations of the celiac axis and the CHA and compared them with those seen in song et $\mathrm{al}^{[6]}$, sureka et $\mathrm{al}^{[17]}$, Osman et al ${ }^{[18]}$ and other studies. Variations in LG, SpA, CHA and SMA were also compared with study done by Ulfacker $\mathrm{s}$ et al. [7]

\section{Interpretation of variation in origin and course of RHA, LHA, MHA and GDA}

After assessing the celiac axis anatomy, we evaluated the CHA anatomy, including its origin site, anatomic course, and relationship to surrounding structures (portal vein or superior mesenteric vein, pancreas head or uncinate process). Next, we evaluated the branching patterns of the downstream hepatic arteriesspecifically, the proper, right, left, and middle hepatic arteries - and the gastroduodenal artery in the patients with celiac axis and CHA variations. Variation in origin and course of RHA, LHA, MHA and GDA were described according to Michel's classification [Table 1], Songs classifycation scheme and Sureka et al. Results were also compared with other studies ${ }^{[2,3,19,20,21,22]}$.

\section{IMA Variants}

In literature, IMA has little variation in terms of position and origin. ${ }^{[23]}$

\section{Renal Arteries Variations}

Renal artery variations were divided into two groups as early branching (EB) into segmental arteries and additional renal artery.

Early branching was defined as branching of the main renal arteries into segmental branches at a more proximal level than the renal hilum or at less than $2 \mathrm{~cm}$ distance from their aortic origin. ${ }^{[24]}$ 
Additional renal artery was more than one main renal artery usually arising from aorta supplying kidney ${ }^{[25]}$. It was also divided into two types-

1. Aberrant renal artery means additional renal artery entering through hilum of kidney.

2. Accessory renal artery means additional renal artery entering kidneys directly piercing poles (inferior/superior) of kidney.

\section{Interpretation of renal artery variation}

First word denotes total number of renal arteries, second one denotes type of additional artery (AC or $A B)$, next is hyphen with further next origin artery of additional artery.
Example- if one kidney was supplied by single renal artery entering through hilum and giving segmental branches at more than $2 \mathrm{~cm}$ distance from their aortic origin, was marked as 1.If one kidney was supplied by single renal artery entering through hilum and giving segmental branches at less than $2 \mathrm{~cm}$ distance from their aortic origin or before renal hilum, was marked as 1E.If one kidney was supplied by main and accessory artery, marked as 2, AC-AORTA. If one kidney was supplied by main and aberrant superior polar artery, marked as 2, AB (SP) AORTA. If two accessory arteries were present, it was marked as 3, AC-AORTA

Table 3. Abbreviation

\begin{tabular}{|c|c|}
\hline Ao & Aorta \\
\hline CA & Celiac axis (hepatogastrosplenic trunk) or its equivalent \\
\hline СH/CHA & $\begin{array}{l}\text { Common hepatic artery, an arterial trunk containing at least one segmental hepatic artery } \\
\text { and the gastroduodenal artery, regardless of its origin site or anatomic course }\end{array}$ \\
\hline CM trunk & Celiacomesenteric trunk \\
\hline GDA & Gastroduodenal artery \\
\hline GMtrunk & Gastromesenteric trunk \\
\hline GSpM trunk & Gastrosplenomesenteric trunk \\
\hline HG trunk & Hepatogastric trunk \\
\hline HGM trunk & Hepatogastromesenteric trunk \\
\hline HGSp trunk & Hepatogastrosplenic trunk, normal celiac axis \\
\hline HM trunk & Hepatomesenteric trunk \\
\hline HSp trunk & Hepatosplenic trunk \\
\hline HSpM trunk & Hepatosplenomesenteric trunk \\
\hline LG & Left gastric artery \\
\hline LH/LHA & Left hepatic artery, which is equivalent to $S 2 / 3 / 4$ \\
\hline HAP & $\begin{array}{l}\text { Proper hepatic artery, which is an arterial trunk before branching into the right and left } \\
\text { hepatic arteries, regardless of its origin site or anatomic course }\end{array}$ \\
\hline RH/RHA & Right hepatic artery, which is equivalent to $\mathrm{S} 5 / 6 / 7 / 8$ \\
\hline SM/SMA & Superior mesenteric artery \\
\hline SpM trunk & Splenomesenteric trunk \\
\hline Sp/SpA/SA & Splenic artery \\
\hline
\end{tabular}




\section{Results and Comparison to other Studies}

Table 4- Celiac axis variations- comparison

\begin{tabular}{|c|c|c|c|c|c|c|}
\hline \multicolumn{2}{|c|}{$\begin{array}{l}\text { Ulfacker's classification }^{7} \\
\quad \text { (only celiac trunk) }\end{array}$} & $\begin{array}{l}\text { Osman et al }^{18} \\
\mathrm{~N}=1000\end{array}$ & $\begin{array}{c}\text { Song's classification } \\
\text { scheme (including celiac } \\
\text { trunk and SMA) }\end{array}$ & $\begin{array}{l}\text { Our study } \\
\mathrm{n}=500(\%)\end{array}$ & $\begin{array}{c}\text { Song's et } \\
\mathrm{al}^{6} \mathrm{~N}=5002\end{array}$ & $\begin{array}{c}\text { Sureka et } \\
\mathrm{al}^{17} \mathrm{~N}=600\end{array}$ \\
\hline \multicolumn{7}{|c|}{ Type } \\
\hline \multirow[t]{4}{*}{$\mathbf{I}$} & Trifurcation & $905(90.5)$ & Normal anatomy & $455(91)$ & \multirow{4}{*}{$4457(89.1)$} & \multirow{4}{*}{$546(91)$} \\
\hline & Classic pattern & $638(63.8)$ & HGSp+SM & $152(30.4)$ & & \\
\hline & Non-classic pattern & $267(26.7)$ & & $285(57)$ & & \\
\hline & Quadfurcation & - & HGSp-RH+SM & $18(3.6)$ & & \\
\hline II & hepato-splenic trunk & $28(2.8)$ & $\mathrm{HSp}$ trunk $+\mathrm{LG}+\mathrm{SM}$ & $17(3.4)$ & $221(4.42)$ & $17(2.83)$ \\
\hline III & hepatogastric trunk & $6(0.6)$ & $\mathrm{HG}$ trunk $+\mathrm{Sp}+\mathrm{SM}$ & 0 & $1(0.02)$ & \\
\hline IV & $\begin{array}{c}\text { hepatospleno-mesenteric } \\
\text { trunk }\end{array}$ & 0 & HSpM trunk + LG & $3(0.6)$ & $34(0.68)$ & $1(0.16)$ \\
\hline \multirow{2}{*}{$\mathbf{V}$} & \multirow{2}{*}{ gastro-splenic trunk } & $43(4.3)$ & $\mathrm{CH}+\mathrm{GSp}$ trunk $+\mathrm{SM}$ & $5(1)$ & $11(0.22)$ & $5(0.83)$ \\
\hline & & & HM trunk + GSp trunk & $5(1)$ & $132(2.64)$ & $4(0.66)$ \\
\hline VI & celiacomesenteric trunk & $6(0.6)$ & $\mathrm{CM}$ trunk & $1(0.2)$ & $53(1.06)$ & $4(0.66)$ \\
\hline VII & celiaco-colic trunk & 0 & Not classified & - & - & - \\
\hline \multirow[t]{9}{*}{ VIII } & no celiac trunk & $10(1)$ & $\mathrm{CH}+\mathrm{LG}+\mathrm{Sp}+\mathrm{SM}$ & $1(0.2)$ & $5(0.10)$ & 0 \\
\hline & & & $\mathrm{HM}$ trunk $+\mathrm{LG}+\mathrm{Sp}$ & $2(0.4)$ & $12(0.24)$ & $2(0.33)$ \\
\hline & & & HG trunk + SpM trunk & $1(0.2)$ & $8(0.16)$ & 0 \\
\hline & & & $\mathrm{CH}+\mathrm{GSpM}$ trunk & 0 & $3(0.06)$ & 0 \\
\hline & & & $\mathrm{CH}+\mathrm{LG}+\mathrm{SpM}$ trunk & 0 & $1(0.02)$ & 0 \\
\hline & & & HSp trunk + GM trunk & 0 & $1(0.02)$ & 0 \\
\hline & & & $\mathrm{HGM}$ trunk $+\mathrm{Sp}$ & 0 & 0 & 0 \\
\hline & & & $\mathrm{CH}+\mathrm{GM}$ trunk $+\mathrm{Sp}$ & 0 & 0 & 0 \\
\hline & & $2(0.2)$ & Ambiguous anatomy & $10(2)$ & $63(1.26)$ & $21(3.5)$ \\
\hline \multicolumn{2}{|r|}{ Bifurcation form } & $77(7.7)$ & - & - & - & - \\
\hline
\end{tabular}

Table 5 CHA Anatomy variations- comparison

\begin{tabular}{|c|c|c|c|c|}
\hline \multicolumn{2}{|c|}{ Anatomic Course and Specific Variation } & $\begin{array}{c}\text { Our study } \\
(\mathrm{n}=500)\end{array}$ & Songs et al $N=4939$ & $\begin{array}{c}\text { Sureka et al } \\
\mathrm{N}=600\end{array}$ \\
\hline \multicolumn{2}{|c|}{ A. Originating from celiac axis or its equivalent } & $476(95.2)$ & $4763(96.44)$ & $575(95.83)$ \\
\hline \multirow[t]{5}{*}{ Suprapancreatic preportal course } & & $474(94.8)$ & $4756(96.29)$ & $576(98.12)$ Total $^{*}$ \\
\hline & HGSp trunk & $453(90.6)$ & $4,443(89.96)$ & \\
\hline & HSp trunk & $17(3.4)$ & $222(4.49)$ & \\
\hline & $\mathrm{CM}$ trunk & 0 & $49(1)$ & \\
\hline & HSpM trunk & $3(0.6)$ & $34(0.7)$ & \\
\hline Originating from left gastric artery & HG trunk & $1(0.2)$ & $8(0.16)$ & \\
\hline Suprapancreatic retroportal course & HGSp trunk & $2(0.4)$ & $6(0.12)$ & 7 (1.19) Total* \\
\hline Transpancreatic pre SMV course & & 0 & 1 & \\
\hline \multicolumn{2}{|c|}{ B. Originating from SMA } & $8(1.6)$ & $148(3.00)$ & $6(1)$ \\
\hline \multirow[t]{3}{*}{ Suprapancreatic preportal course } & & $5(1)$ & $39(0.8)$ & \\
\hline & HM trunk & $4(0.8)$ & $38(0.8)$ & \\
\hline & CM trunk & $1(0.2)$ & 1 & \\
\hline \multirow[t]{3}{*}{ Suprapancreatic retroportal course } & & $3(0.6)$ & $85(1.72)$ & \\
\hline & HM trunk & $3(0.6)$ & $83(1.7)$ & \\
\hline & $\mathrm{CM}$ trunk & 0 & 2 & \\
\hline \multicolumn{2}{|c|}{ C. Originating from aorta: suprapancreatic preportal course } & $6(1.2)$ & $20(0.40)$ & $2(0.33)$ \\
\hline \multicolumn{2}{|c|}{ D. Ambiguous dual pathway: HM trunk } & $1(0.2)$ & 1 & $4(0.66)$ \\
\hline \multicolumn{2}{|l|}{ E. Absent } & $9(1.8)$ & 63() & \\
\hline \multicolumn{2}{|l|}{ F. Not determined } & 0 & - & $13(2.16)$ \\
\hline
\end{tabular}


Table 6 RHA, LHA, GDA, and MHA origins variations and comparison

\begin{tabular}{|c|c|c|c|}
\hline \multicolumn{2}{|c|}{ RHA, LHA, GDA and MHA origins } & $\begin{array}{c}\text { Our study }(\mathrm{N}=500) \\
(\%)\end{array}$ & $\begin{array}{c}\text { Sureka et al. } \\
\mathrm{N}=600\end{array}$ \\
\hline \multicolumn{4}{|c|}{ RHA origin } \\
\hline A. HAP/CHA & & $392(78.4)$ & 478 (79.6) \\
\hline \multirow[t]{4}{*}{ B. $\quad$ Replaced } & & $97(19.4)$ & $91(15.16)$ \\
\hline & SMA & $69(13.8)$ & 81 \\
\hline & Celiac axis & $26(5.2)$ & 8 \\
\hline & Aorta & $2(0.4)$ & 2 \\
\hline \multirow[t]{4}{*}{ C. Accessory } & & $11(2.2)$ & $31(5.16)$ \\
\hline & SMA & $11(2.2)$ & 21 \\
\hline & Celiac axis & 0 & 6 \\
\hline & Aorta & 0 & 4 \\
\hline \multicolumn{4}{|c|}{ LHA origins } \\
\hline A. HAP & & $386(77.2)$ & $489(81.5)$ \\
\hline \multirow[t]{4}{*}{ B. Replaced } & & $30(6)$ & $65(10.8)$ \\
\hline & LGA & $29((5.8)$ & 63 \\
\hline & Aorta & 0 & 2 \\
\hline & $\mathrm{CA}$ & $1(0.2)$ & 0 \\
\hline C. Accessory & LGA & $84(16.8)$ & $46(7.6)$ \\
\hline \multicolumn{4}{|c|}{ GDA origin } \\
\hline A. CHA & & $474(94.8)$ & $586(97.6)$ \\
\hline B. Celiac axis & & $9(1.8)$ & $10(1.6)$ \\
\hline C. RHA & & $13(2.6)$ & $2(0.33)$ \\
\hline D. LHA & & $4(0.8)$ & 0 \\
\hline E. Not defined & & 0 & $2(0.33)$ \\
\hline \multicolumn{4}{|c|}{ MHA origin } \\
\hline A. RHA & & $195(39)$ & $248(41.33)$ \\
\hline B. LHA & & $60(12)$ & $167(27.83)$ \\
\hline C. CHA & & $54(10.8)$ & $27(4.5)$ \\
\hline D. Not defined & & $191(38.2)$ & $158(26.3)$ \\
\hline
\end{tabular}

Table 7 hepatic artery variations in our study-according to Michel's classification

\begin{tabular}{|l|c|}
\hline Michels's & Frequency in our study \\
\hline Type & no $500(\%)$ \\
\hline I & $272(54.4 \%)$ \\
\hline II & $29(5.8 \%)$ \\
\hline III & $69(13.8 \%)$ \\
\hline IV & $5(1 \%)$ \\
\hline V & $84(16.8 \%)$ \\
\hline VI & $11(2.2 \%)$ \\
\hline VII & $4(0.8 \%)$ \\
\hline VIII & $27+1(5.6 \%)$ \\
\hline IX & $8(1.6 \%)$ \\
\hline X & $1(0.2 \%)$ \\
\hline NOD & $6(1.2 \%)$ \\
\hline
\end{tabular}


Table 8 Renal artery variation in our study

\begin{tabular}{|c|c|c|c|}
\hline & $\begin{array}{c}\text { RIGHT KIDNEY } \\
(\mathrm{n}=498)\end{array}$ & $\begin{array}{c}\text { LEFT KIDNEY } \\
(\mathrm{n}=497)\end{array}$ & $\begin{array}{c}\text { Both side } \\
(\mathbf{n}=500)\end{array}$ \\
\hline A. Single renal artery & 428 & 402 & 359 \\
\hline a. $\quad$ Early branching & 53 & 63 & 21 \\
\hline B. Double renal arteries & 64 & 89 & 20 \\
\hline a. Accessory & 53 & 76 & 17 \\
\hline b. $\quad$ Aberrant SP+IP & $8+3=11$ & $10+3=13$ & 0 \\
\hline C. Three renal arteries & 5 & 5 & 0 \\
\hline D. Four renal arteries & 0 & 1 & 0 \\
\hline $\begin{array}{c}\text { E. Horseshow kidneys } \\
\mathrm{N}=2 \text { patients }\end{array}$ & \multicolumn{2}{|c|}{2, each had three arteries } & \\
\hline F. Unilateral kidneys & $\begin{array}{c}\text { 2, each with single } \\
\text { artery }\end{array}$ & $\begin{array}{l}\text { 1, accessory artery } \\
\text { supplying hilum }\end{array}$ & \\
\hline \multicolumn{3}{|c|}{ G. Two renal arteries on the right and three renal arteries on the left } & 2 \\
\hline \multicolumn{3}{|c|}{ H. Two renal arteries on the left and three renal arteries on the right } & 4 \\
\hline \multicolumn{3}{|c|}{ I. Two renal arteries on the right and four renal arteries on the left } & 1 \\
\hline
\end{tabular}

Table. 9 Prevalence of bilateral anomalies of renal arteries in the literature

\begin{tabular}{|l|c|}
\hline Study & Rate of bilateral anomalies \\
\hline Our study & $27 / 500(5.4 \%)$ \\
& Note- if include early branching 48/500 (9.6\%) \\
\hline Saldarriaga et al. [26] & $6 / 194(3.1 \%)$ \\
\hline Kurcz et al. [27] & $7 / 216(3.2 \%)$ \\
\hline Sampaio et al. [28] & $6 / 70(8.57 \%)$ \\
\hline Tarzamni et al. [29] & $11 / 117(9.4 \%)$ \\
\hline Spring et al. [30] & $53 / 444(12 \%)$ \\
\hline Kornafel et al [1] & $20 / 201(10 \%)$ \\
\hline Ugurel et al [7] & $7 / 100(7 \%)$ \\
\hline Basti ram et al [31] & $21 / 200(10.5 \%)$ \\
\hline
\end{tabular}

Range of bilateral extra renal arteries $3.1-12 \%$. Variation in our study stands at $5.4 \%$

Table 10. Renal artery variations- comparison

\begin{tabular}{|c|c|c|c|c|c|c|c|c|c|c|}
\hline & \multicolumn{4}{|c|}{ RIGHT KIDNEY } & \multicolumn{4}{|c|}{ LEFT KIDNEY } & \multicolumn{2}{|c|}{$\begin{array}{c}\text { Both side } \\
\text { (detail on next } \\
\text { table) }\end{array}$} \\
\hline & $\begin{array}{c}A \mathrm{n}=498 \\
(\%)\end{array}$ & B $(\%)$ & C & $\mathrm{D} \mathrm{N}=\mathbf{2 0 0}(\%)$ & $A(n=497)$ & B $(\%)$ & $\mathbf{C}$ & $\begin{array}{c}\mathrm{D} \\
\mathrm{N}=200(\%)\end{array}$ & $A(n=500)$ & $\mathbf{C}$ \\
\hline A. Single renal artery & $428(85.94)$ & $713(83)$ & - & $153(76.5)$ & $402(80.88)$ & $736(86)$ & & $139(69.5)$ & 359 & 62 \\
\hline a. Early branching & $53(10.64)$ & - & 5 & $15(7.5)$ & $63(12.67)$ & - & 7 & $12(6)$ & 21 & 1 \\
\hline B. Double renal arteries & $64(12.8)$ & $126(15)$ & 10 & $32(16)$ & 89 (17.9) & $105(12)$ & 11 & $50(25)$ & 20 & 11 \\
\hline $\begin{array}{ll}\text { a. } & \text { Accessory }\end{array}$ & $53(10.64)$ & - & & $13(6.5)$ & $76(15.29)$ & - & & $15(7.5)$ & 17 & \\
\hline b. Aberrant SP+IP & $\begin{array}{c}8+3=11 \\
(2.2)\end{array}$ & & & $6+13=19(9.5)$ & $10+3=13(2.6)$ & & & $13+22=25$ & 0 & \\
\hline C. $\quad$ Three renal arteries & $5(1)$ & $9(1 \%)$ & 3 & $1(0.5)$ & $5(1)$ & $6(0.7 \%)$ & 2 & 0 & 0 & 1 \\
\hline D. $\quad$ Four renal arteries & $0(0)$ & $0(0 \%)$ & & 0 & $1(0.2)$ & $2(0.2 \%)$ & & & 0 & \\
\hline $\begin{array}{cccc}\text { E. None } & \text { (no } & \text { renal } \\
\text { artery) } & & & \end{array}$ & $1(0.2)$ & $7(0.8 \%)$ & 0 & & $2(0.4)$ & $6(0.7 \%)$ & & & & \\
\hline
\end{tabular}

Note - A. Our study, B. Özkan et $\mathrm{al}^{32}$, C. Kumaresan et $\mathrm{al}^{33}$, D. Kornafel et al. 


\section{JMSCR Vol||06||Issue ||05||Page 576-588||May}

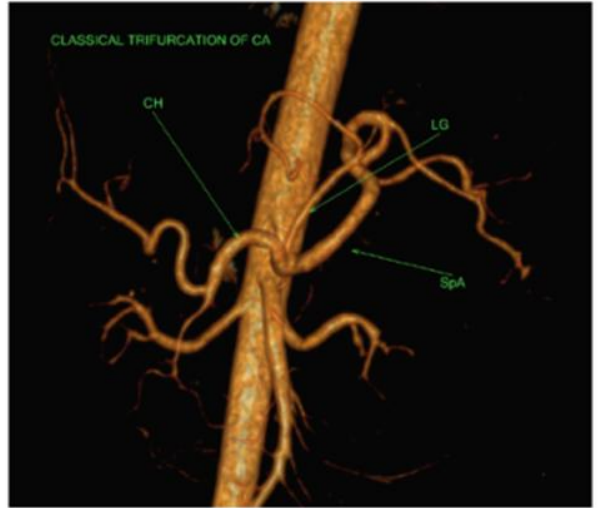

Fig 1a

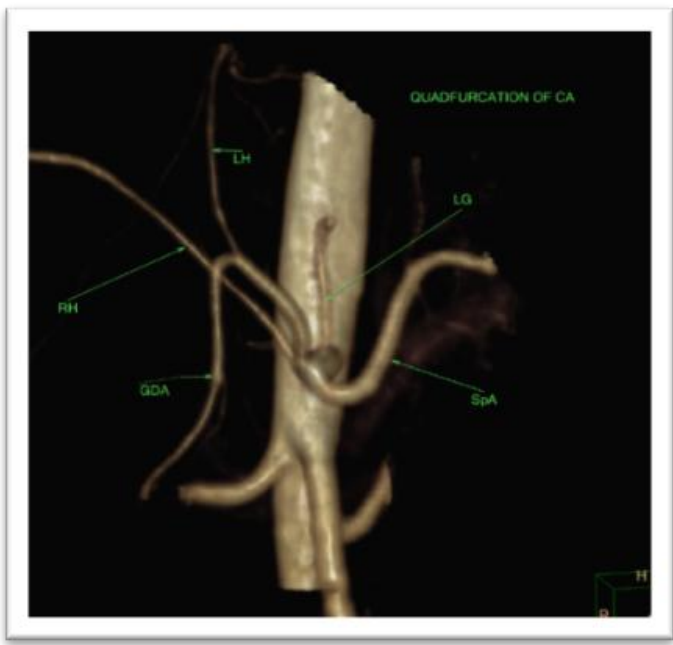

Fig 1c

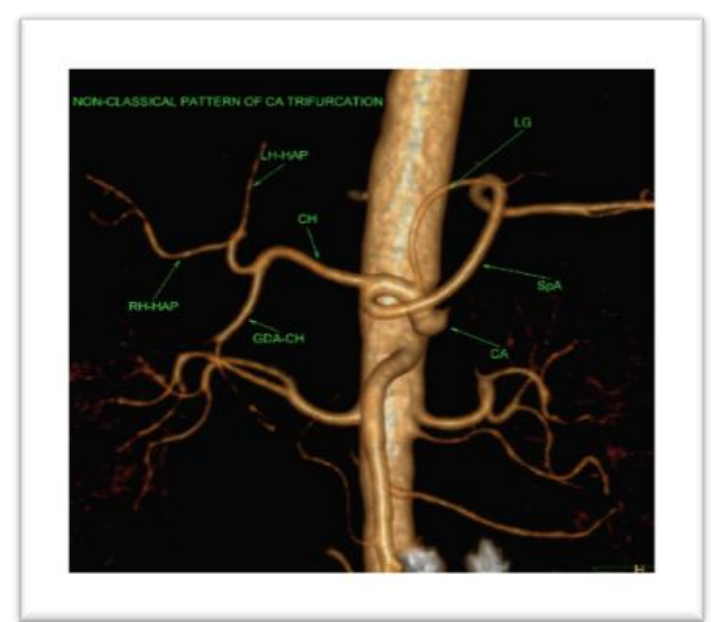

Fig 1b

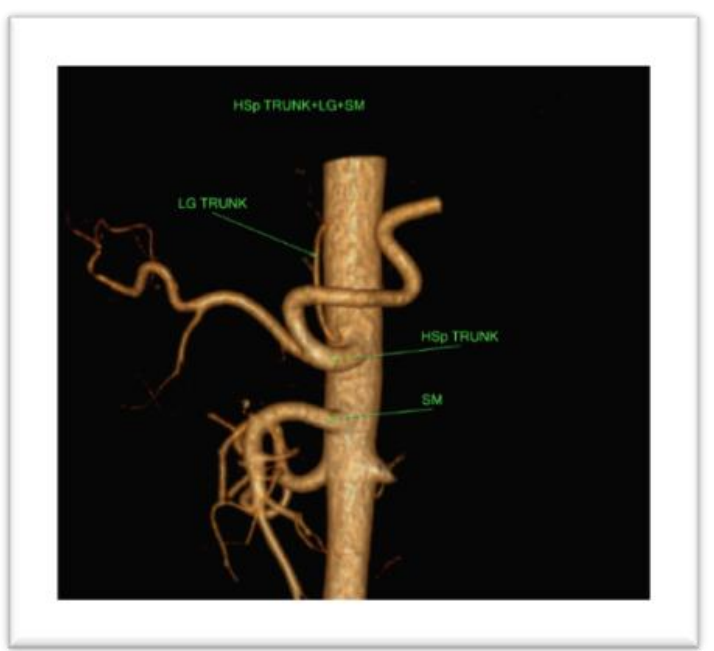

Fig 1d

Fig 1 3D VR images showing a. classical b. non-classical form of normal celiac axis (HGSp trunk + LG) and c. quadfurcation of celiac axis. d. HSp trunk with separate origin of LG and SM from aorta.

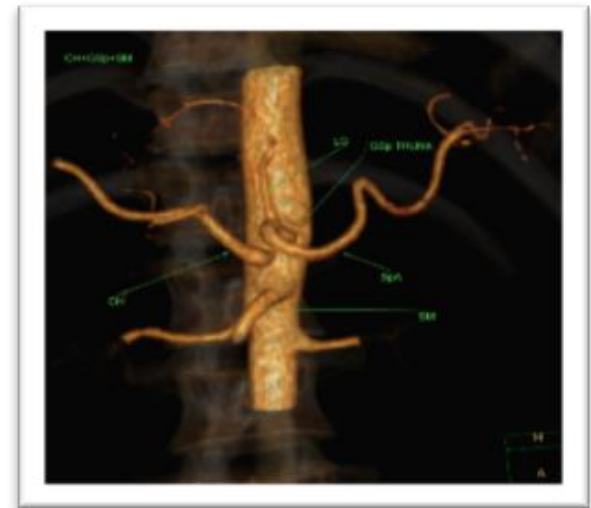

Fig 2A

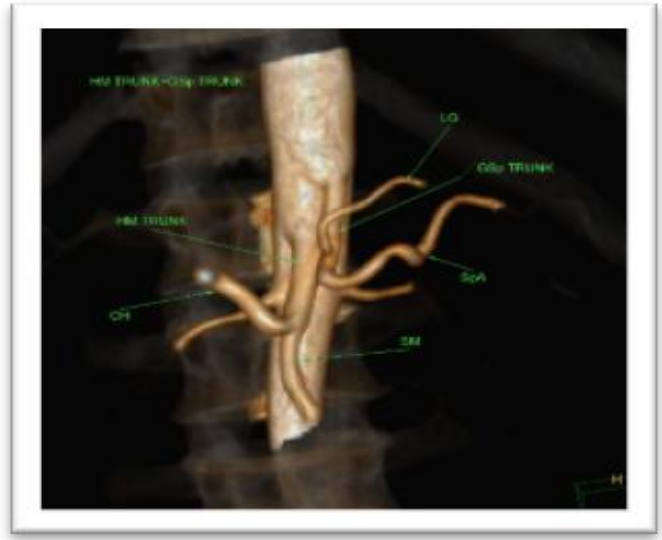

Fig 2B 


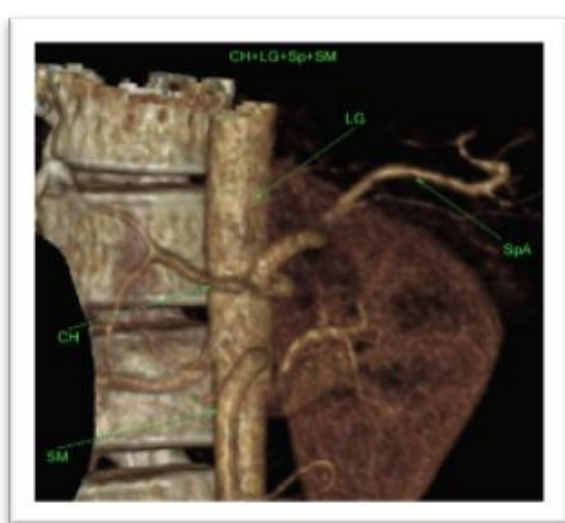

Fig 2C

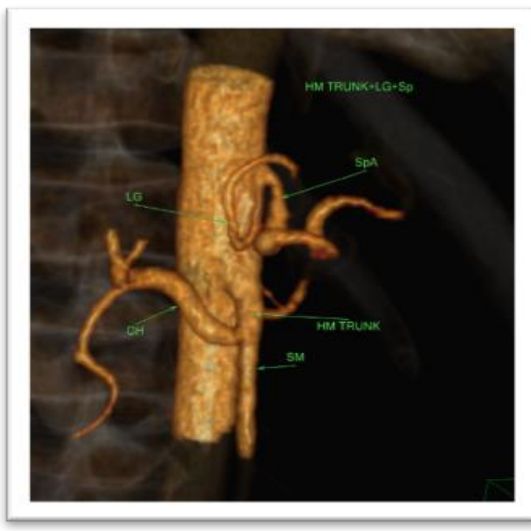

Fig 2D

Fig 2B-D V Rimages showing some of the observed celiac axis variations

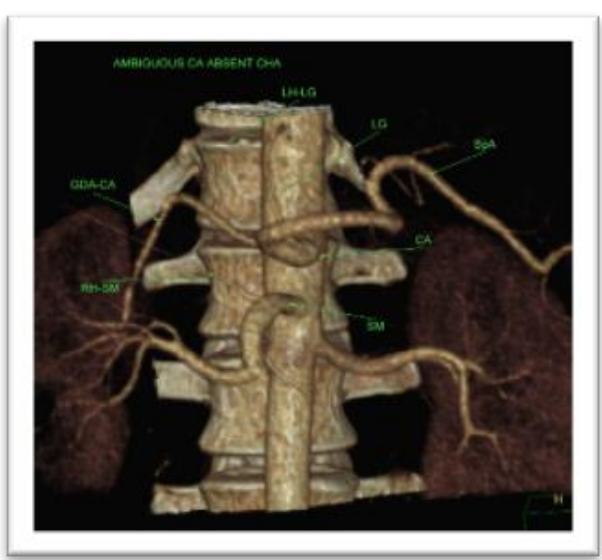

Fig 3A

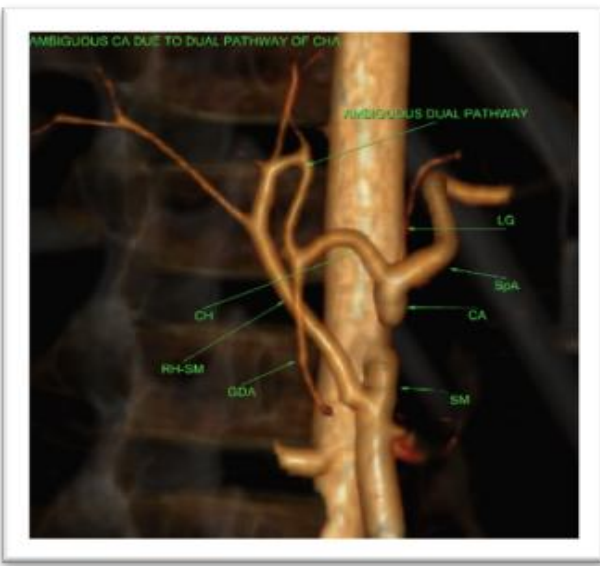

Fig 3B

Fig $3 \mathrm{~A}$ and 3B VR images showingambiguous celiac axis due to absent CHA and ambiguous dual pathway

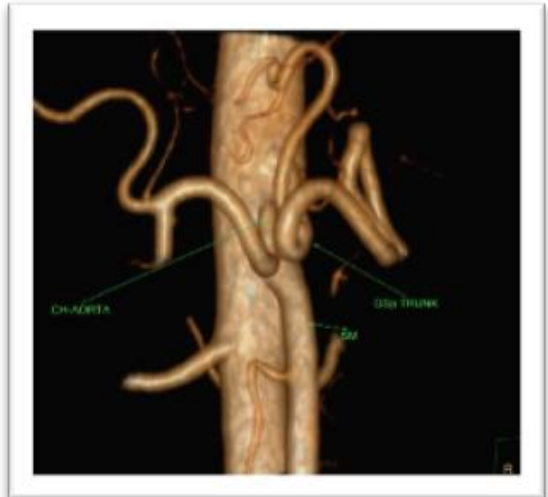

Fig 4 VR image showing replaced origin of CHA from aorta

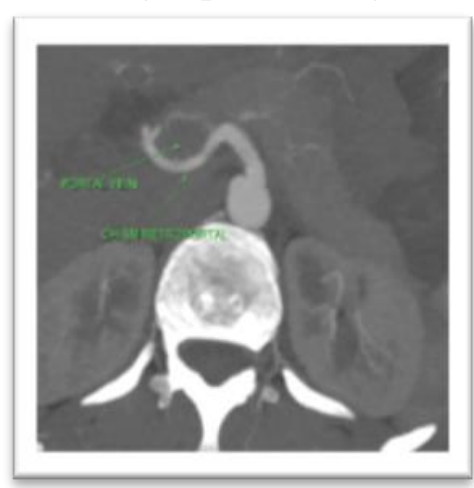

Fig 5 MIP showing suprapancreatic retroportal courseof CHA originating from SMA 


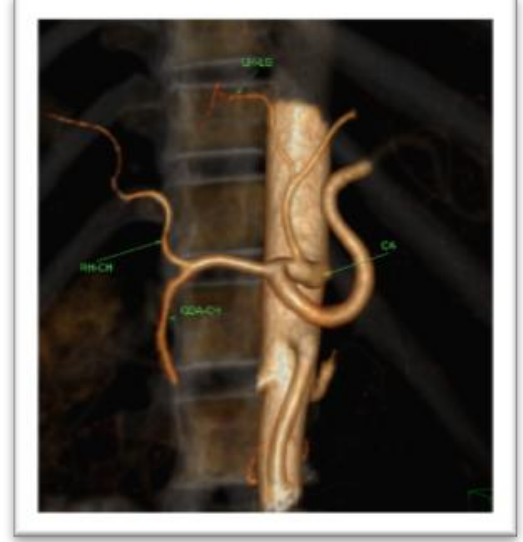

Fig 6A VR

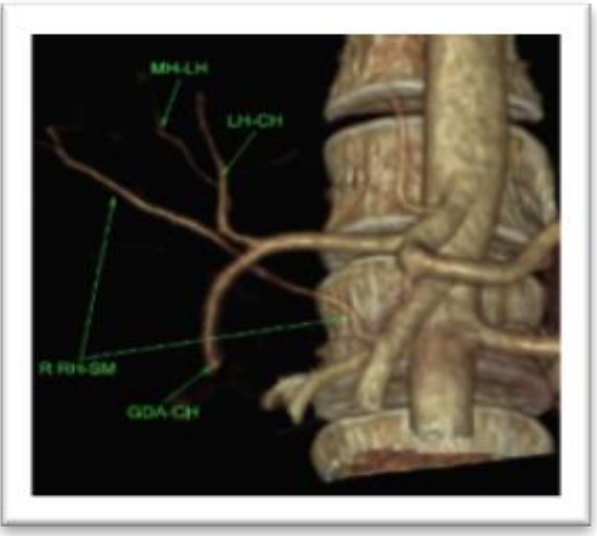

Fig 6B

Fig 6 VR images showing A. replaced LHA from LG and B. replaced RHA from SMA

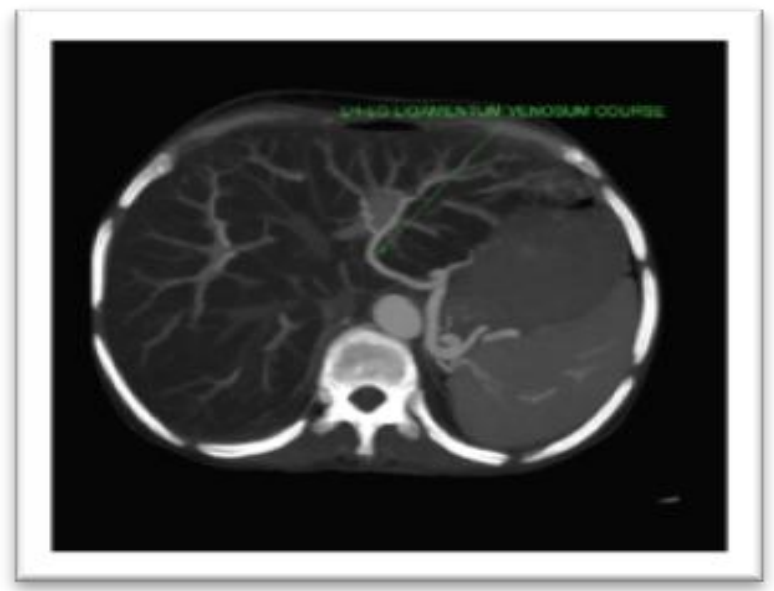

Fig 7 MIP image of showing ligamentum venosum course of LHA

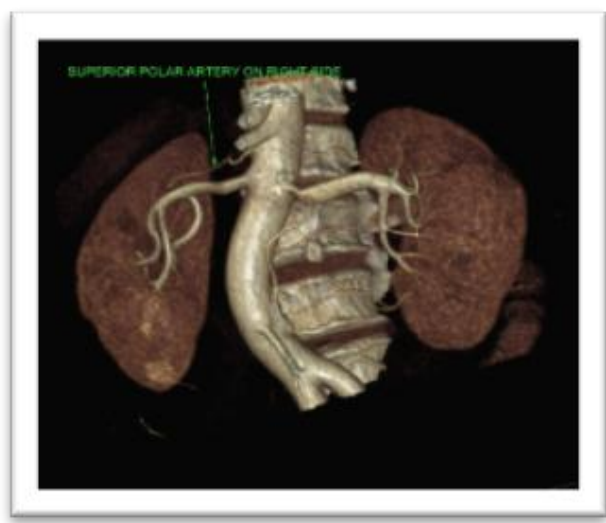

Fig 8 3D VR

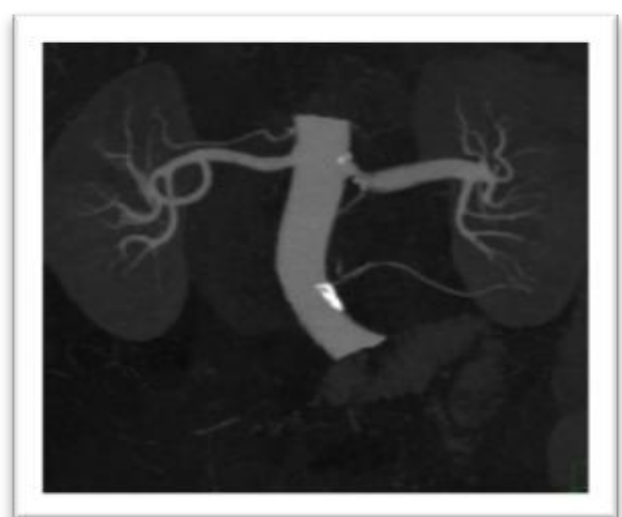

Fig 8 3D MIP

Fig 8 VR and MIP images showing aberrant superior polar renal artery on right side directly entering kidney by piercing renal capsule

\section{Conclusion}

This study is the first of its kind which comprehensively describes variations of celiac axis, hepatic artery branches and renal arteries. Developmental anomalies of the main arteries branching from the abdominal aorta were frequently seen in our study - in 255/500 (51\%) of patients. They were mostly concerning renal arteries and revealing a great variability of variants, with the most common one being the presence of an additional hilar artery. The study showed a statistically significantly higher number 
of vasculature anomalies of the left kidney in comparison to the right kidney. In our study group, renal vasculature anomalies were clearly more frequent in men, but the difference was not statistically significant. No statistically significant association was seen between celiac axis/hepatic artery and renal artery variations.

\section{References}

1. Olga Kornafel, Bogusława Baran, Izabela Pawlikowska, Piotr Laszczyński et al. Analysis of anatomical variations of the main arteries branching from the abdominal aorta, with 64-detector computed tomography. (C) Pol J Radiol, 2010; 75(2): 38-45.

2. Michel NA. Observatons on the blood supply of the liver and gallbladder (200 dissections). Blood supply and anatomy of the upper abdominal organs, with a descriptive atlas. Philadelphia, $\mathrm{Pa}$ : Lippincott ;1955. p. 64-9.

3. Jonathan R. Hiatt, Joubin Gabbay, and Ronald W. Busuttil et al. Surgical Anatomy of the Hepatic Arteries in 1000 Cases. ANNALS OF SURGERY Vol. 220, No. 1, 50-52 C 1994 J. B. Lippincott Company.

4. Vandamme JP, Bonte J, Van der Scheueren G. A reevaluation of hepatic and cystic arteries: The importance of aberrant hepatic branches. ActaAnat 1969;73:192-209.

5. Suzuki T, Nakayasu A, Kawabe K, Takeda $\mathrm{H}$, Honjo I.Surgical signifi cance of anatomic variations of the hepatic artery. Am J Surg 1971;122:505-12.

6. Song SY, Chung JW, Yin YH, Jae HJ, Kim $\mathrm{HC}$, Jeon UB, et al. Celiac axis and common hepatic artery variations in 5002 patients: Systematic analysis with spiral CT and DSA.Radiology 2010;255:278-88.

7. Uflacker R. Atlas of vascular anatomy: an angiographic approach. Baltimore: Williams \& Wilkins, 1997.

8. Catalano OA, Singh AH, Uppot RN, Hahn PF, Ferrone CR, Sahani DV. Vascular and biliary variants in the liver: Implications for liver surgery Radio Graphics 2008;28:35978.

9. Fox M, Yalin R. Renal transplantation with multiple arteries. Br J Urol 1979;51:333-6.

10. Sampaio FJB, Passos MARF. Renal arteries: anatomic study for surgical and radiological practice. Surg Radiol Anat 1992;14:113-17

11. Susan standring. Gray's Anatomy. Churchill Livingstone. 2008;40:1183.

12. Shakeri AB, Tubbs RS, Shoja MM, Pezeshk P, Farahani RM, Khaki AA, et al. Bipolar supernumerary renal artery. Surg Radiol Anat. 2007;29(1):89-92.

13. Rao M, Bhat SM, Venkataramana V, Deepthinath R, Bolla SR. Bilateral prehilar multiple branching of renal arteries: a case report and literature review. Kathmandu University Medical Journal (KUMJ). 2006;4(3):345-48.

14. Satyapal KS, Haffejee AA, Singh B, Ramsaroop L, Robbs JV, Kalideen JM. Additional renal arteries incidence and morphometry. Surg Radiol Anat. 2001;23(1):33-38.

15. Kadir S. Kidneys. In: Kadir S, ed. Atlas ofnormal and variant angiographic anatomy.Philadelphia: W.B. Saunders Company, 1991; 387-429.

16. Boijsen E. Renal angiography: Techniques and hazards; anatomic and physiologic considerations. In: Baum S, ed. Abrams' angiography. 4th ed. Philadelphia: Little, Brown and Company, 1997; 1101-1131.

17. Sureka, et al.: Variations of celiac axis, and its branches in 600 patients- Indian Journal of Radiology and Imaging / August 2013 / Vol 23 / Issue 3.

18. Ahmed M. Osman et al.- Celiac trunk and hepatic artery variants: A retrospective preliminary MSCT report among Egyptian patients. The Egyptian Journal of Radiology and Nuclear Medicine 47 (2016) 1451-1458. 
19. M S Ugurel et al (2010)- Anatomical variations of hepatic arterial system, coeliac trunk and renal arteries: an analysis with multidetector CT angiography. The British Journal of Radiology, 83 (2010), 661-667.

20. De Cecco CN, Ferrari R, Rengo M, Paolantonio P, Vecchietti F, Laghi A. Anatomic variations of the hepatic arteries in 250 patients studied with 64-row CT angiography. EurRadiol. 2009;19(11):2765 -2770. doi: 10.1007/s00330-009-14587. [PubMed] [Cross Ref].

21. Kiat Arjhansiri, Patcharee Charoenrat, Weeranuch Kitsukjit: Anatomic variations of the hepatic arteries in 200 patients done by angiography. publication/ 6122365_2006.

22. Koops A, Wojciechowski B, Broering DC, Adam G, Krupski-Berdien G. Anatomic variations of the hepatic arteries in 604 selective coeliac and superior mesenteric angiographies. Surg Radiol Anat2004;26:239-44 [PubMed].

23. Richard D. White et al- The Celiac Axis Revisited: Anatomic Variants, Pathologic Features, and Implications for Modern Endovascular Management. Radio Graphics 2015; 35:879-898.

24. Gumus H, Brdal Ozdemir E, Cetincakmak ML, Tekbas G, Ekici, Onder H, et al. Variations of renal artery in 820 patients using 64-detector CT-Angiography. Renal Faliure. 2012;34:286-90.

25. Rubin GD, Alfrey EJ, Dake MD, et al. Assessment of living renal donors with spiral CT. Radiology 1995; 195:457-462.

26. Saldarriaga B, Pérez AF, Ballesteros LE: A direct anatomical study of additional renal arteries in a Colombian mestizo population. Folia Morphol, 2008; 67(2): 129-34.
27. Kurcz J, Nienartowicz E, Słonina J et al: The usefulness of CT-angiography in detecting anatomical variants of arteries arising from the abdominal aorta and aortic arch. Adv Clin Exp Med, 2007: 16(6): 75160 .

28. Sampaio FJB, Passos MA: Renal arteries: anatomic study for surgical and radiological practice. Surg Radiol Anat, 1992; 14: 11317

29. Tarzamni MK, Nezami N, Rashid RJ et al: Anatomical differences in the right and left renal arterial patterns. Folia Morphol, 2008; 67(2): 104-10.

30. Spring DB, Salvatierra O Jr, Palubinskas $\mathrm{AJ}$, et al. Results and significance of angiography in potential kidney donors. Radiology 1979; 133:45-47.

31. Basti Ram S et al. - Spectrum of coeliac trunk, hepatic artery and renal artery variations- analysis by multidetector computed tomography. International Journal of Recent Trends in Science and Technology, ISSN 2277-2812 E-ISSN 2249-8109, Volume 12, Issue 1, 2014 pp 168-172.

32. Ozkan U, Oguzkurt L, Tercan $\mathrm{T}$ et al : Renal artery origins and variations: angiographic evaluation of 855 consecutive patients. Diagn Interv Radiol, 2006; 12: 183-86.

33. Kumaresan Munnusamy et al (2016). Variations in Branching Pattern of Renal Artery in Kidney Donors Using CT Angiography. J Clin Diagn Res. 2016 Mar; 10(3): AC01-AC03. Published online 2016 Mar 1. doi: 10.7860/JCDR/2016/ 16690.7342 . 